\title{
Is the European System for Cardiac Operative Risk Evaluation model valid for estimating the operative risk of patients considered for percutaneous aortic valve replacement?
}

\author{
Morgan L. Brown, MD, ${ }^{a}$ Hartzell V. Schaff, MD, ${ }^{a}$ Maurice E. Sarano, MD, ${ }^{b}$ Zhuo Li, MS, ${ }^{c}$ Thoralf M. Sundt, MD,
} Joseph A. Dearani, MD, ${ }^{a}$ Charles J. Mullany, MBMS, and Thomas A. Orszulak, MD ${ }^{a}$

From the Divisions of Cardiovascular Surgery, ${ }^{\mathrm{a}}$ Cardiovascular Medicine, ${ }^{\mathrm{b}}$ Biostatistics, ${ }^{\mathrm{c}}$ Mayo Clinic, Rochester, Minn.

Received for publication Oct 4, 2007; accepted for publication Oct 30, 2007.

Address for reprints: Hartzell V. Schaff, MD, Mayo Clinic, 200 1st St SW, Rochester MN 55905 (E-mail: schaff@mayo.edu).

J Thorac Cardiovasc Surg 2008;136:566-71 $0022-5223 / \$ 34.00$

Copyright $(0) 2008$ by The American Association for Thoracic Surgery

doi:10.1016/j.jtcvs.2007.10.091
Objective: The European System for Cardiac Operative Risk Evaluation has been used to define a particularly high-risk group of patients for aortic valve replacement in whom alternative procedures, such as stent-mounted percutaneous valve procedures, may be appropriate. Our objective was to assess the validity of this risk assessment at a large-volume, tertiary cardiac surgical center.

Methods: From January 1, 2000, to December 30, 2006, a total of 1177 patients underwent isolated aortic valve replacement at the Mayo Clinic. Patient and operative demographics were recorded in a prospective database. Early mortality ( $\leq 30$ days) was obtained. Additive and logistic European System for Cardiac Operative Risk Evaluations were calculated for each patient.

Results: The mean patient age was 68.0 years ( \pm 14.7 years) at the time of surgery, and $36.8 \%$ were female. Variables used in the calculation of the European System for Cardiac Operative Risk Evaluation included chronic lung disease (15\% of our cohort), extracardiac arteriopathy $(13.8 \%)$, neurologic dysfunction $(0.2 \%)$, previous cardiac surgery $(23.2 \%)$, renal failure $(6.5 \%)$, active endocarditis $(3.1 \%)$, recent myocardial infarction $(1.1 \%)$, unstable angina $(0.1 \%)$, and severe pulmonary hypertension $(6.5 \%)$. The ejection fraction was severely reduced $(\leq 30 \%)$ in $4.9 \%$ of patients and moderately reduced $(\leq 50 \%)$ in $12.7 \%$ of patients. One percent of patients were in a critical state, and operation was performed urgently in 3.4\% of patients. Although mean mortality estimates were $6.9 \% \pm 3.4 \%$ (additive European System for Cardiac Operative Risk Evaluation) and 10.9\% $\pm 12.7 \%$ (logistic European System for Cardiac Operative Risk Evaluation), actual overall operative mortality in our patients was 2.5\%. Additive and logistic European System for Cardiac Operative Risk Evaluations overestimated operative mortality in low, intermediate, and high-risk subgroups by up to $17.8 \%$.

Conclusions: The European System for Cardiac Operative Risk Evaluation should not be used to determine the operability of patients for isolated aortic valve replacement. Elevated European System for Cardiac Operative Risk Evaluations alone do not appropriately define a population for use of a percutaneous aortic valve.

$\mathrm{R}$ isk scoring systems are tools to enable the comparison of patients' risks. Risk scores may be used for operative scheduling (urgency of operation), patient care requirements and dismissal planning, and auditing of mortality and quality of care. ${ }^{1}$ However, scoring systems should not be used as substitutes for clinical decision making. ${ }^{1}$ An ideal scoring system should be easy to use, have a clearly defined outcome, and be used for auditing purposes. ${ }^{2}$ 


\section{Abbreviations and Acronyms}

AVR = aortic valve replacement

CABG = coronary artery bypass grafting

EuroSCORE $=$ European System for Cardiac

Operative Risk Evaluation

The introduction of novel technologies, such as percutaneous aortic valves, poses a significant ethical challenge because, at early stages of development, the results obtained may be anticipated to be suboptimal. Their use is appropriately restricted to extremely high-risk groups for whom conventional procedures may be prohibited or unavailable. Although highly sophisticated surgical risk-scoring systems have been developed for purposes of quality assurance, they are currently being used to define such groups.

The European System for Cardiac Operative Risk Evaluation (EuroSCORE) is a risk scoring system designed to predict operative mortality in cardiac surgical patients. It was designed because of the increasing numbers of high-risk patients undergoing cardiac surgery and a desire to provide riskadjusted mortality rates instead of only crude mortality rates. ${ }^{3}$ The additive EuroSCORE was the first model available for use. ${ }^{3}$ However, because of concerns regarding the underestimation of mortality in high-risk subsets, the logistic model was made available. ${ }^{4,5}$ The additive model is still considered adequate in most cases and has the advantage of being easily calculated at the bedside.

Despite the limitations of scoring systems, investigators studying new technologies such as percutaneous aortic valves have implied that EuroSCORE may be a tool to identify patients at excessive operative risk for a traditional aortic valve replacement (AVR). ${ }^{6-8}$ To test this theory, we reviewed AVRs performed at the Mayo Clinic and compared our outcomes with the predictive additive and logistic EuroSCORE scoring systems.

\section{Materials and Methods}

Institutional review board approval was obtained for this study. From January 1, 2000, to December 30, 2006, a total of 1177 patients underwent isolated AVR at the Mayo Clinic. Patients with concomitant procedures including coronary artery bypass grafting $(\mathrm{CABG})$ and ascending aortic procedures were excluded. Patient and operative demographics were recorded in a prospective database according to the Society of Thoracic Surgeons Adult Database definitions. Early mortality ( $\leq 30$ days) was obtained for all patients using hospital records and the Accurint database (www.accurint.com).

The EuroSCORE research calculator available online was used for both additive and logistic EuroSCORE calculations. The EuroSCORE considers 17 risk factors, including patient, cardiac, and operative features, to estimate operative mortality. ${ }^{9}$ In the risk models, patient characteristics associated with increased perioperative mortality include older age, female gender, chronic pulmonary disease, extracardiac arteriopathy, neurologic disease, previous cardiac sur- gery, elevated serum creatinine, active endocarditis, and a critical preoperative state (Table 1). ${ }^{5}$ Cardiac-related factors include unstable angina requiring intravenous nitrates, left ventricular dysfunction, recent myocardial infarction, and pulmonary hypertension. Operation-related factors include emergency surgery, other procedures than isolated $\mathrm{CABG}$, surgery on the thoracic aorta, and postinfarction septal rupture. ${ }^{1,9}$ Both additive and logistic EuroSCOREs were calculated for each patient.

The definitions in the Society of Thoracic Surgery Adult Cardiac Surgery Database were matched exactly to those in the EuroSCORE models whenever possible. Extracardiac arteriopathy was defined as peripheral vascular disease, which included claudication, either with exertion or rest, amputation for arterial insufficiency, aortoiliac occlusive disease reconstruction, peripheral vascular bypass surgery, angioplasty, or stent, documented abdominal aneurysm or repair/ stent, or documented positive noninvasive testing. Patients who had a history of cerebrovascular disease were also included (unresponsive coma $>24$ hours, cerebrovascular event, transient ischemic attack, noninvasive carotid test with $>75 \%$ occlusion, or previous carotid surgery). Patients who had a recent cerebral vascular accident $(<72$ hours) were considered to have neurologic disease. Previous cardiac surgery requiring opening of the pericardium included only those who had previous surgery using cardiopulmonary bypass. Those patients in cardiogenic shock (systolic blood pressure $<80 \mathrm{~mm} \mathrm{Hg}$ and/or cardiac index $<1.8$ despite maximal treatment or intravenous inotropes and/or intra-aortic balloon pump necessary to maintain blood pressure $>80$ and/or cardiac index $>1.8$ ) were defined as having a critical preoperative state. All patients in this study had an isolated AVR and thus had an "operation other than isolated coronary artery bypass grafting (CABG)."

Patient characteristics were entered into the EuroSCORE additive and logistic models. The calculated scores were used as predictors for operative mortality ( $<30$ days) in a logistic regression model. Receiver operating curves were created for the overall group comparing both additive and logistic models. Patients were then divided into low-risk (additive $\leq 5$, logistic $\leq 0.05$ ), intermediate-risk (additive 6-10, logistic 0.051-0.10), and high-risk (additive $\geq 11$, logistic $\geq 0.101$ ) groups, and the observed and expected number of events for these risk groups were compared using chi-square or Fisher exact tests as appropriate. Analysis was performed using SAS 9.1 (SAS Inc, Cary, NC).

\section{Results}

The mean age at the time of AVR was 68 years $( \pm 14.7$ years), and $37 \%$ were female. Fifteen percent of patients had chronic lung disease, $13.8 \%$ of patients had extracardiac arteriopathy, $0.2 \%$ of patients had neurologic dysfunction, $23.2 \%$ of patients had previous cardiac surgery, $6.5 \%$ of patients had renal failure, $3.1 \%$ of patients had active endocarditis, $1.1 \%$ of patients had a recent myocardial infarction, $0.1 \%$ of patients had unstable angina, $1.1 \%$ of patients had recent myocardial infarction, and $6.5 \%$ of patients had severe pulmonary hypertension. The ejection fraction was severely reduced $(\leq 30 \%)$ in $4.9 \%$ of patients and moderately reduced $(\leq 50 \%)$ in $12.7 \%$ of patients. One percent of patients were in a critical state, and urgent surgery was performed in $3.4 \%$ of 
TABLE 1. Additive and logistic EuroSCORE definitions and calculations

\begin{tabular}{|c|c|c|c|}
\hline Patient-related factors & Definition & Additive score & Logistic score \\
\hline Age & Per 5 y or part thereof $>60 y$ & 1 & 0.0666354 \\
\hline Sex & Female & 1 & 0.3304052 \\
\hline Chronic pulmonary disease & $\begin{array}{l}\text { Long-term use of bronchodilators or steroids } \\
\text { for lung disease }\end{array}$ & 1 & 0.4931341 \\
\hline Extracardiac arteriopathy & $\begin{array}{l}\text { Any one or more of the following: claudication, carotid } \\
\text { occlusion, or }>50 \% \text { stenosis, previous or planned } \\
\text { intervention on the abdominal aorta, limb arteries } \\
\text { or carotids }\end{array}$ & 2 & 0.6558917 \\
\hline $\begin{array}{l}\text { Neurologic dysfunction } \\
\text { disease }\end{array}$ & Severely affecting ambulation or day-to-day functioning & 2 & 0.841626 \\
\hline Previous cardiac surgery & $\begin{array}{l}\text { Any previous cardiac surgery requiring opening the } \\
\text { pericardium }\end{array}$ & 2 & 1.002625 \\
\hline Serum creatinine & $>200 \mu \mathrm{mol} / \mathrm{L}$ preoperatively & 2 & 0.6521653 \\
\hline Active endocarditis & $\begin{array}{l}\text { Patient still receiving antibiotic treatment for } \\
\text { endocarditis at the time of surgery }\end{array}$ & 3 & 1.101265 \\
\hline Critical preoperative state & $\begin{array}{l}\text { Any one or more of the following: ventricular tachycardia, } \\
\text { fibrillation, or aborted sudden death, preoperative cardiac } \\
\text { massage, preoperative ventilation before arrival to the OR, } \\
\text { preoperative inotropic support, intra-aortic balloon } \\
\text { counterpulsation, or preoperative acute renal failure } \\
\text { (anuria or oliguria }<10 \mathrm{~mL} / \mathrm{h} \text { ) }\end{array}$ & 3 & 0.9058132 \\
\hline \multicolumn{4}{|l|}{ Cardiac-related } \\
\hline Unstable angina & $\begin{array}{l}\text { Rest angina requiring IV nitrates until arrival in the } \\
\text { anesthetic room }\end{array}$ & 2 & 0.5677075 \\
\hline \multirow[t]{2}{*}{ LV dysfunction } & Moderate (EF 30\%-50\%) & 1 & 0.4191643 \\
\hline & poor $(\mathrm{EF}<30 \%)$ & 3 & 1.094443 \\
\hline Recent myocardial infarction & $<90 \mathrm{~d}$ before surgery & 2 & 0.5460218 \\
\hline Pulmonary hypertension & Systolic PA pressure $>60 \mathrm{~mm} \mathrm{Hg}$ & 2 & 0.7676924 \\
\hline \multicolumn{4}{|l|}{ Operation-related factors } \\
\hline Emergency & OR before the beginning of the next working day & 2 & 0.7127953 \\
\hline Other than isolated CABG & $\begin{array}{l}\text { Major cardiac procedures other than or } \\
\text { in addition to CABG }\end{array}$ & 2 & 0.5420364 \\
\hline Surgery on thoracic aorta & Ascending, arch, or descending aorta & 3 & 1.159787 \\
\hline Postinfarction septal rupture & Ventricular septal rupture postinfarction & 4 & 1.462009 \\
\hline Intercept value & For logistic model only & NA & -4.789594 \\
\hline
\end{tabular}

$O R$, Operating room; $I V$, intravenous; $L V$, left ventricular; $E F$, ejection fraction; $P A$, pulmonary artery; $C A B G$, coronary artery bypass grafting.

patients. All patients had a procedure other than CABG (ie, AVR), and no patients had an aortic aneurysm repair or postinfarction ventricular septal defect. This is shown in Table 2 along with the patient characteristics from the original EuroSCORE model.

The area under the curve for the receiver operating curve was 0.75 for the additive score and 0.75 for the logistic score, with $P$ values of less than .001 (Figure 1). This indicated that both additive and logistic EuroSCORE models were reasonably good predictors of operative mortality for the whole group. When the receiver operating curve area under the curve was compared between the additive and logistic EuroSCOREs, there was no significant difference $(P=.329)$. The observed operative mortality ( $<30$ days) was $2.5 \%$ (30/1177 patients). The actual and estimated mortalities are shown in Figure 2.

\section{Additive EuroSCORE}

In the low-risk group (additive EuroSCORE $\leq 5$ ), the estimated mean mortality was $3.5 \% \pm 1.2 \%$, compared with the observed mortality of 3 of 435 patients $(0.7 \%)(P=$ .004 ) (Figure 3, $A$ ). In the intermediate-risk group (additive EuroSCORE 6-10), the estimated mean mortality was $7.7 \% \pm 1.4 \%$, compared with the observed mortality of 14 of 567 patients $(2.5 \%)(P<.001)$. The high-risk group (additive EuroSCORE $\geq 11$ ) had an estimated mean mortality of $12.6 \% \pm 2.0 \%$, compared with the observed mortality of 13 of 175 patients $(7.4 \%)(P=.109)$.

\section{Logistic EuroSCORE}

In the low-risk group (logistic EuroSCORE $\leq 0.05$ ), the estimated mean mortality was $2.8 \% \pm 1.1 \%$, compared with the observed mortality of 3 of 491 patients $(0.6 \%)$ 
TABLE 2. Patient characteristics

\begin{tabular}{lcc}
\hline \multicolumn{1}{c}{ Parameter } & $\begin{array}{c}\text { EuroSCORE } \\
\text { N = 19,030 }\end{array}$ & $\begin{array}{c}\text { Mayo isolated } \\
\text { AVR N = 1177 }\end{array}$ \\
\hline Mean age ( \pm SD) y & $62.5 \pm 10.7$ & $68.0 \pm 14.7$ \\
Female (\%) & 27.8 & 36.8 \\
Chronic pulmonary disease (\%) & 3.9 & 15.0 \\
Extracardiac arteriopathy (\%) & 11.3 & 13.8 \\
Neurologic dysfunction (\%) & 1.4 & 0.2 \\
Previous cardiac surgery (\%) & 7.3 & 23.2 \\
Serum creatinine & 1.8 & 6.5 \\
$\quad>$ 200 mmol/L (\%) & 1.1 & \\
Active endocarditis (\%) & 4.1 & 3.1 \\
Critical preoperative state (\%) & 25.6 & 1.0 \\
LVEF 30\%-50\% (\%) & 5.8 & 12.7 \\
LVEF <30\% (\%) & 9.7 & 4.9 \\
Recent myocardial & & 1.1 \\
$\quad$ infarction (\%) & 2.0 & 6.5 \\
Pulmonary hypertension (\%) & 4.9 & 3.4 \\
Emergency (\%) & 36.4 & 100 \\
Other than isolated CABG (\%) & 2.4 & 0 \\
Surgery on thoracic aorta (\%) & 0.2 & 0 \\
Postinfarction septal rupture (\%) & & \\
\hline EurSCORE, Europan Systm & &
\end{tabular}

EuroSCORE, European System for Cardiac Operative Risk Evaluation; AVR, aortic valve replacement; $S D$, standard deviation; $L V E F$, left ventricular ejection fraction; $C A B G$, coronary artery bypass grafting.

$(P=.007)$ (Figure $3, B)$. In the intermediate-risk group (logistic EuroSCORE 0.051-0.10), the estimated mean mortality was $7.1 \% \pm 1.5 \%$, compared with the observed mortality of 4 of 283 patients $(1.4 \%)(P<.001)$. In the high-risk group (logistic EuroSCORE $\geq 0.101$, the estimated mean mortality was $23.6 \% \pm 14.8 \%$, compared with the observed mortality of 23 of 399 patients $(5.7 \%)(P<.001)$.

\section{Discussion}

With the advent of less-invasive technologies, physicians look to models of operative risk to identify patients who are expected to have high periprocedural mortality. In recent reports of percutaneous and transapical AVR, investigators have used additive and logistic EuroSCOREs as evidence of the high operative risk with a traditional AVR in these patients. ${ }^{6-8,10-13}$ Our study demonstrates that both the additive and logistic EuroSCORE models substantially overestimate mortality in all risk subgroups at a large-volume referral center.

In 1999, the results of the EuroSCORE was published using information on risk factors and mortality on 19,030 patients who underwent cardiac surgery using cardiopulmonary bypass from September to December of 1995 in 128 European centers. $^{3} \mathrm{~A}$ total of 68 preoperative and 29 operative factors were collected, and a logistic regression model was used to estimate operative mortality. Although this study has been validated in the CABG population, ${ }^{14,15}$ there were only 3200 patients who underwent AVR $(\sim 17 \%)$ in the original cohort of 19,030 patients. As a consequence, the models are

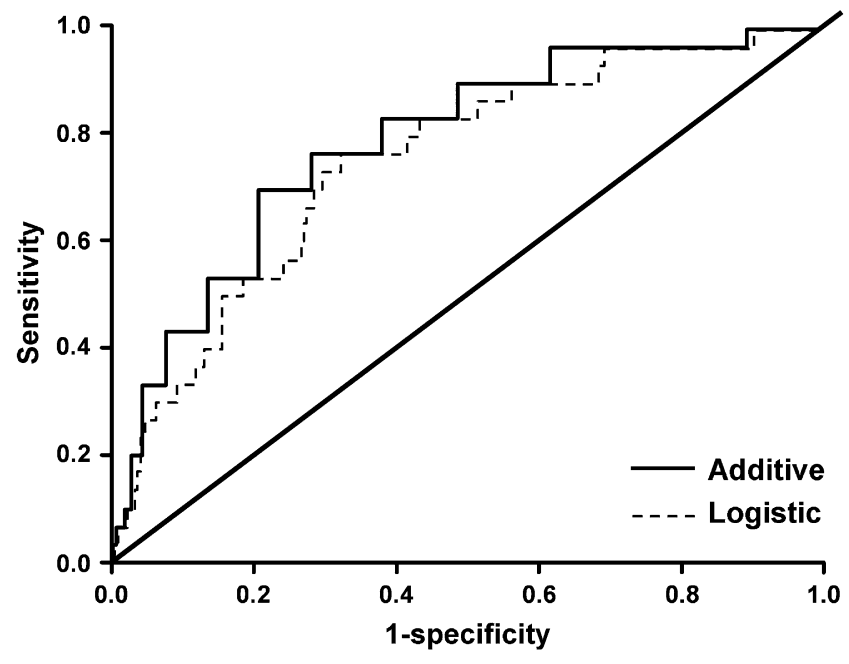

Figure 1. Receiver operator curve of the additive and logistic EuroSCORE models compared with the actual mortality after AVR.

designed so that $\mathrm{CABG}$ is considered the baseline risk and any valve procedure is designated as a "procedure other than CABG." Thus, mitral valve repair, mitral valve replacement, AVR, or any of the previous procedures with concomitant bypass grafting will result in the same EuroSCORE risk profile. Therefore, the relatively lower risk procedures, such as an isolated AVR, may result in an "elevated" risk prediction in both EuroSCORE models.

The Society of Thoracic Surgeons reported an operative mortality of approximately $3.5 \%$ in 2006 for an isolated

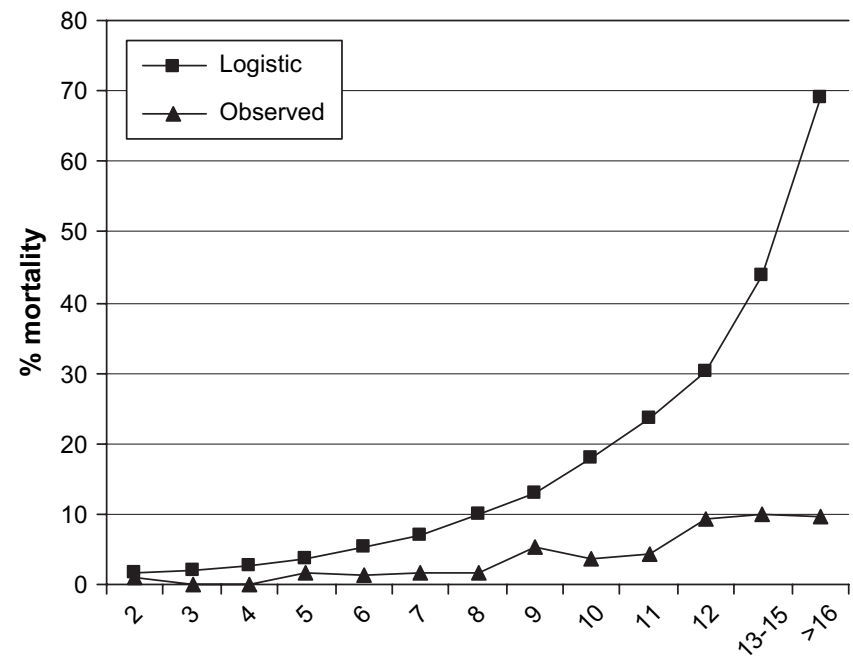

Estimated Additive EuroSCORE

Figure 2. Actual and predicted mortality for the logistic EuroSCORE model. The mortality rate is on the $Y$ axis, and patients are divided into groups on the basis of the predicted additive EuroSCOREs on the $X$ axis. 


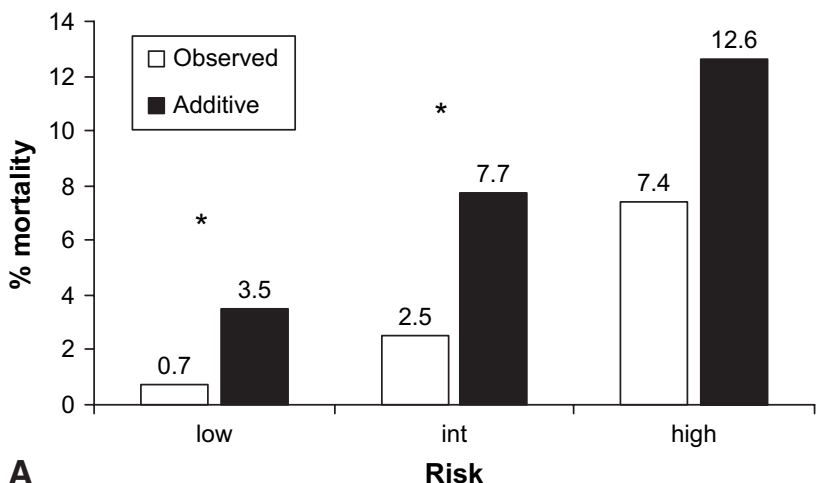

A

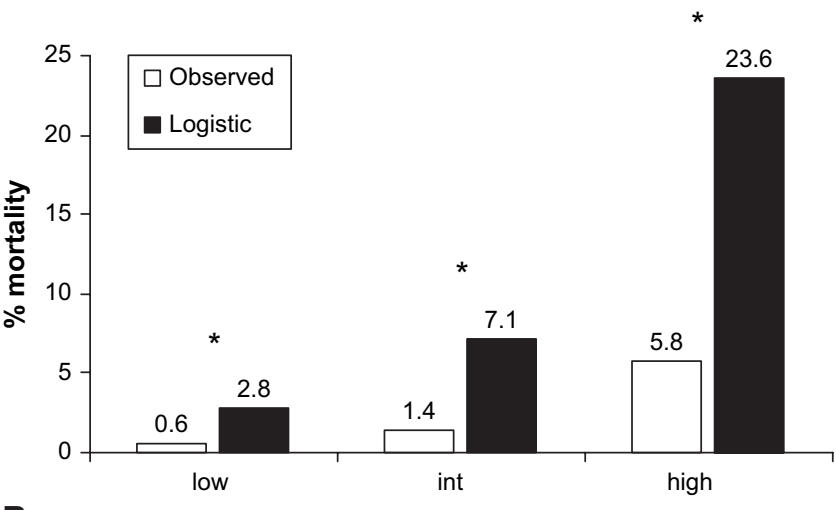

B

Risk

Figure 3. A, Additive EuroSCORE predicted mortality versus observed mortality in risk subgroups. Patients were divided into risk groups on the basis of additive EuroSCORE (low $\leq 5$, intermediate 6-10, high $\geq 11$ ). B, Logistic EuroSCORE predicted mortality versus observed mortality in risk subgroups. Patients were divided into risk groups on the basis of logistic EuroSCORE (low $\leq 0.05$, intermediate $0.051-0.10$, and high $\geq 0.101$ ). *Statistically significant difference. Int, Intermediate.

AVR. ${ }^{16}$ Our overall institutional mortality was only $2.5 \%$ despite that $23 \%$ of patients had at least one previous cardiac procedure requiring cardiopulmonary bypass and $18 \%$ of patients had a reduced left ventricular ejection fraction. We believe that there are few true contraindications to traditional AVR in patients with severe aortic valve stenosis. Most patients who do not undergo AVR are unlikely to be candidates for any interventional procedure because of comorbidities such as dementia or cancer.

The definition of "inoperability" is difficult, as evidenced by the "conversion" of such patients to open traditional procedures after percutaneous misadventures. In the study by Grube and colleagues, ${ }^{7} 6$ "inoperable" patients required urgent conversion to an open procedure to retrieve the devices and implanted aortic prostheses with only 1 operative death. In the series from Vancouver reported by Webb and colleagues, ${ }^{11} 1$ patient had successful balloon dilatation but unsuccessful deployment of an aortic valve. This patient had successful elective AVR 4 months later. Thus, although these patients were considered high risk by EuroSCORE criteria, they underwent successful traditional AVR, which questions their "inoperable" designation.

Our study is limited because the definitions recorded in our databases do not exactly match those in the EuroSCORE risk calculators. The definitions provided by EuroSCORE are broad, especially neurologic disease dysfunction, which is defined as "severely affecting ambulation or day-to-day functioning." This could be interpreted multiple ways. In our study, we limited this to patients who had a stroke within the last 72 hours. We included only patients who had prior cardiopulmonary bypass as "prior cardiac surgery," and only patients in cardiogenic shock were considered as "critical preoperative state." As such, we think our definitions were conservative.

\section{Conclusions}

Less-invasive aortic valve procedures will undoubtedly find a place within current cardiac surgical practice. However, clinicians must be cautious in estimating operative risk from models that were not intended for this specific use. In our practice, both additive and logistic EuroSCORE risk models overestimate operative mortality for isolated AVR, and this overestimation is greatest in high-risk patients.

\section{References}

1. Hariharan S, Zbar A. Risk scoring in perioperative and surgical intensive care patients: a review. Curr Surg. 2006;63:226-36.

2. Ramsay G, MacGregor JR, Murray GD, et al. Prediction of surgical risk in adults. Surg Res Comm. 1988;3:9-103.

3. Roques F, Nashef SA, Michel P, et al. Risk factors and outcome in European cardiac surgery: analysis of the EuroSCORE multinational database of 19030 patients. Eur J Cardiothorac Surg. 1999;15:816-22.

4. Roques F, Michel P, Gladstone AR, Nashef SAM. The logistic EuroSCORE. Eur Heart J. 2003;24:881-2.

5. Zingone B, Pappalardo A, Dreas L. Logistic versus additive EuroSCORE. A comparative assessment of the two models in an independent population sample. Eur J Cardiothorac Surg. 2004;26:1134-40.

6. Ye J, Cheung A, Lichtenstein SV, et al. Six-month outcome of transapical transcatheter aortic valve implantation in the initial seven patients. Eur J Cardiothorac Surg. 2007:31:16-21.

7. Grube E, Schuler G, Buellesfeld L, et al. Percutaneous aortic valve replacement for severe aortic stenosis in high-risk patients using the second- and current third- generation self-expanding CoreValve prosthesis. J Am Coll Cardiol. 2007;50:69-76.

8. Grube E, Laborde JC, Gerckens U, et al. Percutaneous implantation of the CoreValve self-expanding valve prosthesis in high-risk patients with aortic valve disease. Circulation. 2006;114:1616-24.

9. The EuroSCORE research calculator. Available at: http://euroscore.org/ Euroscore\%20Research\%2030.xls. Accessed August 28, 2007.

10. Chandavimol M, McClure SJ, Carere RG, et al. Percutaneous aortic valve implantation: a case report. Can J Cardiol. 2006;22:1159-61.

11. Webb JG, Chandavimol M, Thompson CR, et al. Percutaneous aortic valve implantation retrograde from the femoral artery. Circulation. 2006;113:842-50.

12. Lichtenstein SV, Cheung A, Ye J, et al. Transapical transcatheter aortic valve implantation in humans: initial clinical experience. Circulation. 2006;114:591-6. 
13. Walther T, Falk V, Borger MA, et al. Minimally invasive transapical beating heart aortic valve implantation-proof of concept. Eur J Cardiothorac Surg. 2007;31:9-15.

14. Sergeant P, de Worm E, Meyns B. Single centre, single domain validation of the EuroSCORE on a consecutive sample of primary and repeat CABG. Eur J Cardiothorac Surg. 2001;20:1176-82.
15. Nilsson J, Algotsson L, Hoglund P, Luhrs C, Brandt J. Early mortality in coronary bypass surgery: the EuroSCORE versus The Society of Thoracic Surgeons risk algorithm. Ann Thorac Surg. 2004;77:1235-9.

16. Fall 2006 STS Executive Report. Available at: http://www.sts.org/ documents/pdf/STS-ExecutiveSummaryFall2006-REVISED.pdf. Accessed August 1, 2007.

\section{AATS Academy \\ Friday, May 8, 2009 • Boston, Massachusetts, USA (immediately preceding the $89^{\text {th }}$ Annual AATS Meeting)}

\section{Announcing the new AATS Academy, an intensive didactic and interactive program open to surgeons showing significant promise as potential future division chiefs or who have recently assumed the role of division chief.}

GOALS

- To provide participants with the administrative, interpersonal, mentoring and negotiating skills necessary to serve successfully as a division chief.

- To provide an opportunity for participants to be introduced to well known thoracic surgical leaders who might subsequently serve as personal mentors.

\section{CANDIDATE QUALIFICATIONS}

- Nominated by division chief/department chair/AATS member/self nomination

- Achieved the rank of Associate Professor

- Active/successful clinical surgeon

- Evidence of academic productivity including peer-reviewed publications and presentations at regional or national scientific meetings.

Visit www.aats.org for the complete listing of qualifications, faculty and application information. Applications must be submitted electronically.

APPLICATION DEADLINE: December 1, 2008

Academy participants will be selected by the Academy Committee and notified by February 6, 2009. 